\title{
Hepatobiliary Schistosomiasis
}

\author{
Yehia Shaker*, Nervana Samy and Esmat Ashour \\ Biochemistry Department, National Research Center, Cairo, Egypt
}

\begin{abstract}
Schistosomiasis is an ancient parasitic disease that has afflicted Egyptians since the time of the pharaohs. The disease is caused by lodged schistosome eggs in the host liver, evoking an immune response and leading in some patients to the development of hepatic granuloma and fibrosis. Here, we review the epidemiology, immunopathogenesis, and clinical profile of schistosomiasis. This information may aid in the development of more efficacious treatments and improved disease prognosis.

(c) 2014 The Second Affiliated Hospital of Chongqing Medical University. Published by XIA \& HE Publishing Ltd. All rights reserved.
\end{abstract}

\section{Introduction}

Of the more than 207 million people infected with schistosomiasis, $85 \%$ live in Africa. An estimated 700 million additional people are at risk of infection in 76 countries where the disease is considered endemic due to exposure to infested water agriculturally, domestically, or recreationally. ${ }^{1}$ Worldwide, 200,000 deaths are attributed to schistosomiasis annually. ${ }^{2}$ There is a long history in Egypt of diseases caused by schistosomiasis, notably hematuria. This symptom classically appeared in young boys and was at one time deemed a sign of puberty. Schistosomiasis, sometimes referred to as bilharzia, bilharziasis, or snail fever, was discovered in 1851 by Theodore Bilharz, a German surgeon working in Cairo. He identified the etiological agent as Schistosoma hematobium. ${ }^{4}$ Most schistosomiasis is caused by $S$. haematobium, $S$. mansoni, and S. japonicum. Less prevalent species include S. mekongi and S. intercalatum, which may also cause systemic human disease.

When schistosoma eggs are trapped in tissues, they activate an immunologic reaction and induce schistosomiasis. Antigens released from the egg stimulate a granulomatous reaction involving T cells, macrophages, and eosinophil that cause clinical disease. Symptoms and signs depend on the number and location of eggs. The initial inflammatory

Keywords: Schistosomiasis; Immunopathogenesis; Hepatosplenic schistosomiasis; Co-infection and complications; Anti-schistosomal vaccine

Abbreviations: APC, antigen-presenting cells; CCA, circulating cathodic antigen; DSRS, distal splenorenal shunts; EGDS, esophagogastric devascularization with splenectomy; HBsAg, hepatitis B surface antigen; HBV, hepatitis B virus; HCV, hepatitis C virus; HIV, human immunodeficiency virus; HSS, hepatosplenic schistosomiasis; IL, interleukin; MHC, major histocompatibility complex; SRS, splenorenal shunt.

Received: 9 June 2014; Revised: 23 July 2014; Accepted: 24 July 2014 DOI: $10.14218 /$ JCTH.2014.00018.

*Correspondence to: Yehia Shaker, Biochemistry Dept, National Research Centre, Dokki, Giza 12622, Egypt. Email: ymshaker@gmail.com reaction is readily reversible. However, as the disease progresses, there is collagen deposition and fibrosis, resulting in organ damage that may be only partially reversible. ${ }^{5}$

\section{Life cycle of parasite}

The three species of schistosomes that commonly affect humans (S. hematobium, S. mansoni and S. japonicium) have similar life cycles and develop by a succession of stages: the egg, miracidium, first stage sporocyst, second stage sporocyst, cercariae, schistosomule, and adult. All three species of schistosomes infect in the same manner: direct contact with surface water containing free-living form known as cercariae, which can penetrate the skin. Schistosome ceracariae consist of a tail (used for motility in the water), a head region (used for attachment to host skin), and glands that contain proteolytic enzymes to facilitate penetration of the skin.

In addition to several other major changes, the tail of the cercariae is shed during penetration of the skin to yield a new form called the schistosomulum. After penetrating the wall of a nearby vein, schistosomula are carried in the host blood flow, eventually reaching the portal venous system. It is here where they grow and reach sexual maturity, the mature male and female worms pair, and then, depending on species, migrate to the vessels of the bowel or bladder for egg production. Many eggs pass through the intestinal or bladder wall and are excreted in the feces or urine. The schistosome lifecycle is completed when the eggs hatch, releasing freeswimming miracidia that can in turn re-infect freshwater snails.

However, it is the eggs, not the worms, that cause the pathophysiology of schistosomiasis, and some eggs remain lodged in the tissues of the host. In intestinal schistosomiasis, eggs lodged in the mucosa or submucosa of the gut cause granulomatous reactions, which may then extend into the gut lumen as pseudopapillomas. These pseudopapillomas can cause colonic obstruction and blood loss. Eggs residing in the liver may cause portal fibrosis, leading to portal hypertension, splenomegaly, and esophageal and gastric varices. Exsanguination by esophageal variceal bleeding is the major cause of death by schistosomes. ${ }^{7}$

\section{Immunopathogenesis}

Hepatosplenic schistosomiasis (HSS) is due to inflammation and fibrosis in the presinusoidal portal areas of the liver. Investigations in an experimental murine model have unequivocally shown that the egg stage of the parasite $s$ responsible for these pathological changes. ${ }^{8}$

Shortly after developing into schistosomula, the parasites shed surface antigens and acquire blood group glycolipid and major histocompatibility complex (MHC) antigens from the 
host. Since they express host antigens on their tegument, the schistosomes resemble the host tissue and can thereby avoid attack by the immune system. Although the host suffers little damage directly from the adult worms, the eggs elicit an intense granulomatous inflammatory response. The egg granulomas are well-circumscribed aggregates of inflammatory cells composed of eosinophils, lymphocytes, macrophages, and neutrophils that are embedded in a collagenized extracellular matrix. Hepatic granuloma formation in $S$. mansoni infections is mediated by $\mathrm{MHC}$ class 11 -restricted CD4+ Th cells. ${ }^{9}$

During the early stages of granuloma formation, the immune system initiates a Th-1 type response to schistosomal egg antigens. ${ }^{10}$ The proinflammatory cytokines produced by the activated antigen-presenting cells (APC) stimulate the Th-1 lymphocytes to secrete interferon gamma and interleukin (IL)-2, which in turn further stimulate the APC. Subsequently, with the aid of B7 (mostly B7-2) co-stimulatory signaling and the cytokines IL-4 and IL-10, there is a switch from a Th-1 to a Th-2 response. The Th- 2 response is characterized by increased levels of the anti-inflammatory cytokines IL-4, IL-5, IL-10, and IL-13, antibody production by B cells, and recruitment of eosinophils. Eosinophils generate IL-4, which serves to secure the Th-2 immune response and to induce APCs with alternative activation and secretion profiles. The hepatic egg granulomas that form during the Th-2 immune response appear to be beneficial by limiting the spread of egg secretions and causing little or no parenchymal inflammation of the liver. In contrast, granulomas formed in Th-1 environment are less efficient and can be accompanied by severe hepatocellular microvesicular change, inflammation, and necrosis. ${ }^{11}$

A well-known phenomenon, originally described in the mouse, is the progressive downsizing of granuloma formation in response to eggs arriving in the liver after the period of acute disease. An important mechanism in the immunomodulatory process may involve the anti-inflammatory cytokine $\mathrm{K}-10$, which can inhibit accessory cell function and inflammatory cytokine production. ${ }^{12}$

\section{Clinical manifestation}

Disease syndromes associated with schistosomiasis include cercarial dermatitis, larval pneumonitis, acute schistosomiasis (Katayama fever), and HSS.

\section{Acute schistosomiasis}

In a previously uninfected person, Katayama fever will develop in about 4 to 6 weeks. The onset of symptoms coincides with the initiation of egg deposition. Katayama fever has been described in people with initial heavy infections caused by $S$. mansoni, S. haematobium, and especially S. japonicum. It is believed to represent a serum sickness-like reaction that results from circulating immune complexes. Symptoms include fever, chills, arthralgias, myalgias, dry cough, wheezing, abdominal pain, and diarrhea. Physical findings include rash, tender hepatomegaly splenomegaly, and lymph- adenopathy. Peripheral eosinophilia and high levels of immunoglobulin (Ig)G and IgE are usually present. Symptoms will usually resolve spontaneously after several weeks in most patients. ${ }^{13}$

\section{Hepatosplenic schistosomiasis}

The pathology of hepatic schistosomiasis was first described in Egypt by the great British pathologist Symmers (1904) before either its life cycle or clinical aspects were understood. He found that HSS with portal hypertension and esophageal varices was due to a presinusoidal block of portal blood flow and increased flow from the spleen. ${ }^{14}$

While all schistosome species may cause some degree of hepatobiliary disease, S. hematobium is less likely to do so, and affects primarily the urinary tract. The main species that cause hepatobiliary disease are S. mansoni and S. japonicum. Intestinal and hepatosplenic syndromes may overlap to some degree, but in most cases one or the other prevails. Severe HSS is likely due to failure of immunomodulation processes. Predictably, severely affected patients exhibit a Th- 1 types cytokine profile with little if any detectable IL-10. Conversely, the mild intestinal form of schistosomiasis is associated with a Th-2 cytokine profile. ${ }^{15}$ Symptoms of HSS include hepatomegaly, hepatic fibrosis, portal hypertension, and, with chronic infection, portosystemic collateral blood flow. As the infection progresses, splenomegaly, esophagogastric varices, and hemorrhoids develop. Bleeding from esophageal varices leads to hematemesis and melena. Patients with HSS generally tolerate variceal bleeding episodes better than patients with portal hypertension associated with viral or alcoholic hepatitis. A feature characteristic of HSS is the maintenance of liver perfusion by an increase in hepatic arterial flow, allowing for the preservation of hepatocyte function in the absence of hepatotropic viral coinfections. Neither the eggs nor the adult form of S. mansoni cause damage to hepatocytes. ${ }^{16}$

In the compensated stage of HSS, patients present with predominantly left hepatic lobe enlargement and moderate splenomegaly. Laboratory findings include normal transaminases, mildly elevated alkaline phosphatase, increased IgG, leukopenia, mild hemolytic anemia, and thrombocytopenia. The decompensated phase of HSS is characterized by a shrunken liver, splenomegaly, esophageal varices, hepatic encephalopathy, massive ascites, hypoalbuminemia, and muscle wasting. There is a remarkable lack of other stigmata of chronic liver disease such as jaundice, spider nevi, palmar erythema, testicular atrophy, and gynecomastia. Patients generally do not develop cirrhosis unless they are co-infected with another hepatotropic organism. ${ }^{16,17}$ HSS should be considered in any patient without stigmata of cirrhosis, from an endemic area, who presents with splenomegaly, portal hypertension, and variceal bleeding. ${ }^{18}$

\section{Co-infections and complications}

Co-infection of hepatitis, HIV, and malaria with schistosomiasis can increase the risk for hepatocellular carcinoma and mortality. Patients with schistosomiasis have higher rates of hepatitis B surface antigen ( $\mathrm{HBsAg}$ ) carriage and hepatitis C virus (HCV) seropositivity than non-infected controls. This association may be due, at least in part, to transmission of hepatitis viruses via contaminated needles during blood transfusion and parenteral therapy for schistosomiasis. Schistosomiasis potentiates hepatitis B virus (HBV) and $\mathrm{HCV}$ infections by prolonging hepatic inflammation after an episode of acute viral hepatitis and by increasing the risk of chronic infection. ${ }^{19}$ 


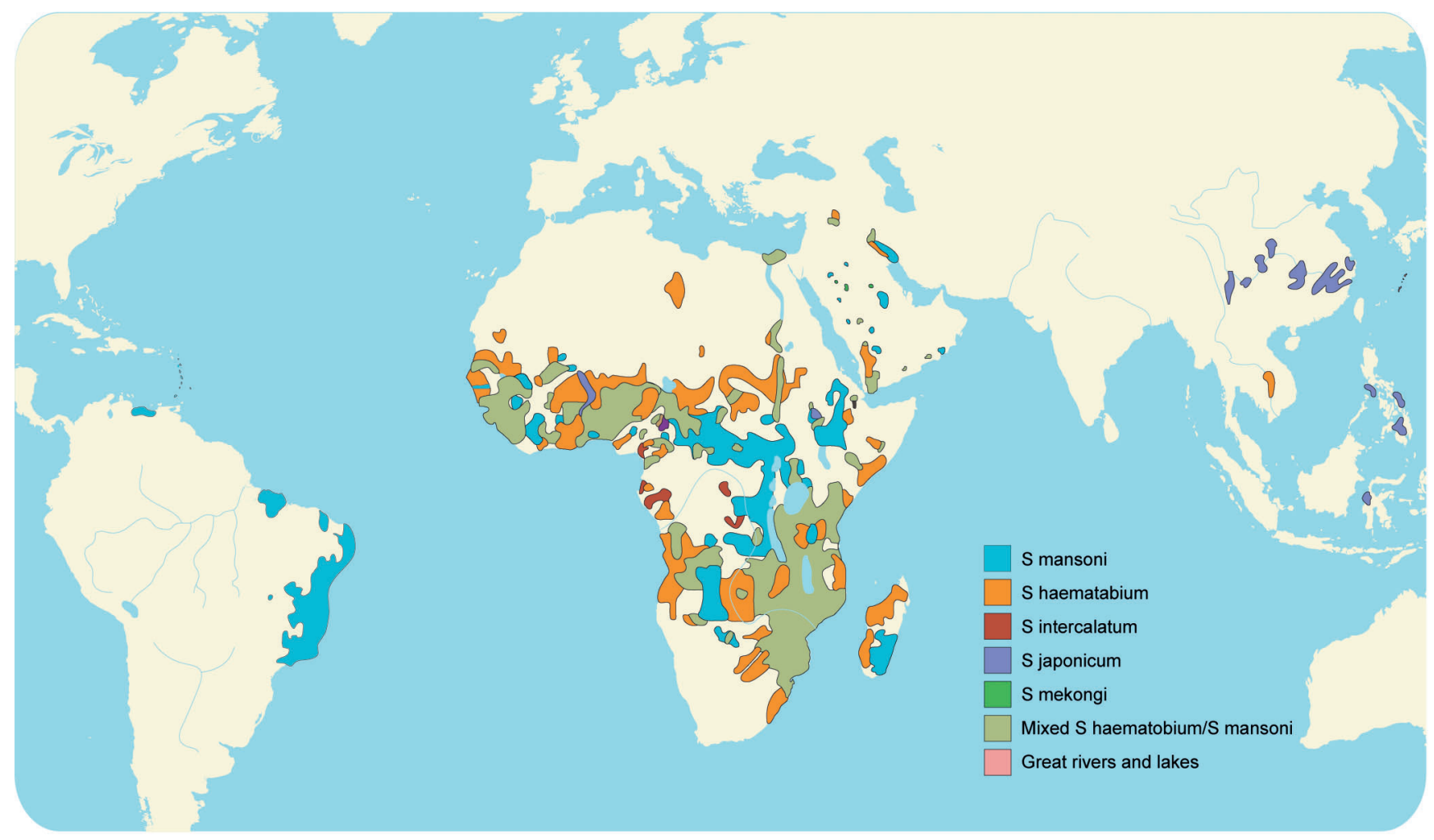

Fig 1. Global distribution of schistosomiasis. Adapted from Gryseels et al. ${ }^{3}$

Increased levels of aminotransferases and bilirubin associated with histological and periportal inflammation on liver biopsy have been reported in patients with HSS and HBV or $\mathrm{HCV}$ co-infections. In addition, liver function deteriorates more rapidly, often with the development of severe, irreversible periportal fibrosis and a more rapid progression toward end-stage liver disease. Development of hepatocellular carcinoma has also been reported in schistosomiasis. ${ }^{20}$ However, some studies have failed to demonstrate a definitive relationship between chronic hepatitis B or C infection and positive ultrasonography or histologic evidence of schistosomal hepatic fibrosis. Potential explanations for these discrepancies include false-positive anti-HCV tests secondary to anti-schistosomal antibodies, small sample size, study design, short duration of follow-up, and bias in recruitment, selection, or observation of patients. ${ }^{21}$

One way to prevent exacerbation of liver disease in schistomiasis with co-infection of hepatitis B is immunization against HBV. Schistosome-infected school children appear to respond well to the hepatitis $B$ vaccine, but hepatomegaly or splenomegaly can significantly hamper sero-conversion. Therefore, vaccination in early childhood should be universal in countries endemic for the hepatotropic schistosomes. ${ }^{22}$

Because of the increasing prevalence of immunodeficiency virus (HIV) infection in many countries that are highly endemic for schistosomiasis, the interaction between these two infectious diseases is being monitored closely. A recent study from Kenya found significantly lower fecal egg counts in subjects with HIV infection, and a direct correlation between the CD4+ T-cell count and the level of egg excretion. No difference, however, was seen in plasma levels of circulating cathodic antigen (CCA), a direct measure of the worm burden, between HIV-infected and non-HIV patients with $S$. mansoni infection. The therapeutic response, as measured by a reduction of fecal egg excretion and schitosomal CAA levels, to standard doses of praziquantel in HIV-seropositive subjects was found to be equal to non-infected controls. Clearly, given the large overlap in their geographic distribution, further studies are needed regarding the interactions between HIV and schistosomiasis. ${ }^{23}$

Although no clear link has been made between S. mansoni and cancer, epidemiologic studies in China and Japan have implicated S. japonicum infection as a risk factor for both primary hepatocellular carcinoma and colorectal cancer. Risk factors for the development of liver cancer, including viral coinfection with HBV or HCV and alcohol abuse, are usually present as well. ${ }^{2}$

\section{Prognosis}

HSS carries a relatively good prognosis because hepatic function is preserved until the end of the disease (unless variceal bleeding occurs). Although treatment is indicated for patients with end-stage complications of portal hypertension and severe pulmonary hypertension, these patients are not likely to benefit from it. Indeed, cor pulmonale usually does not improve significantly with treatment. Three operations have been used for the successful treatment of portal hypertension due to schistosomiasis: esophagogastric devascularization with splenectomy (EGDS), distal splenorenal 

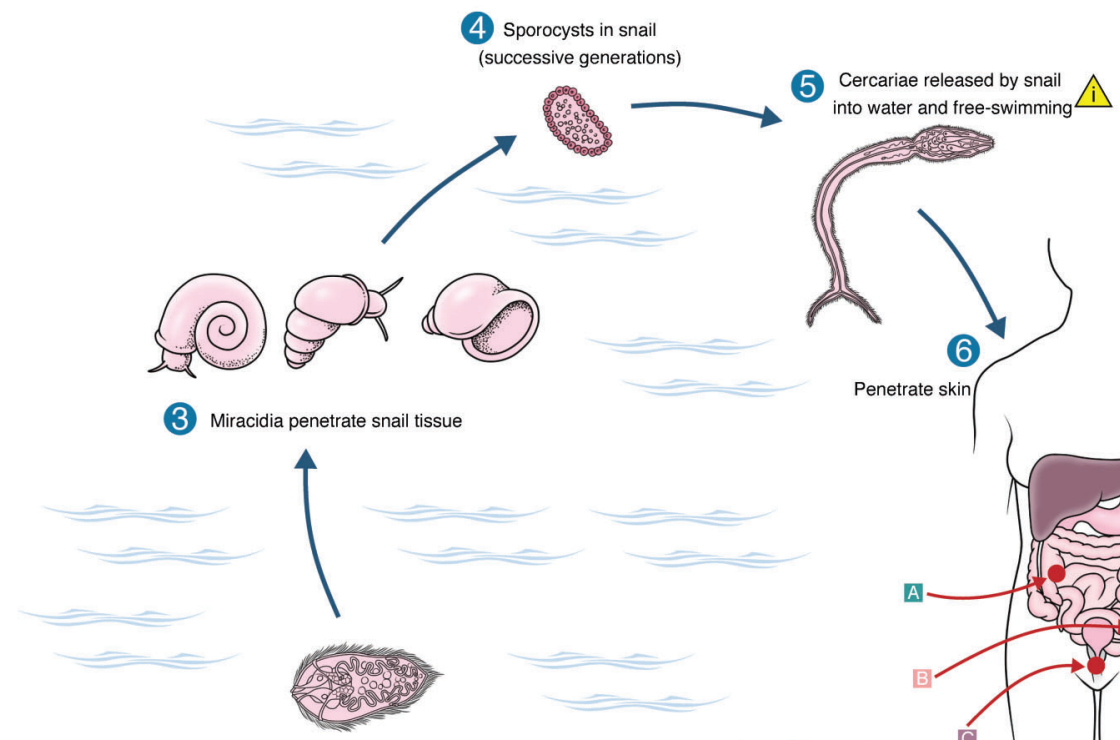

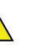

d $=$ Diagnostic Stage
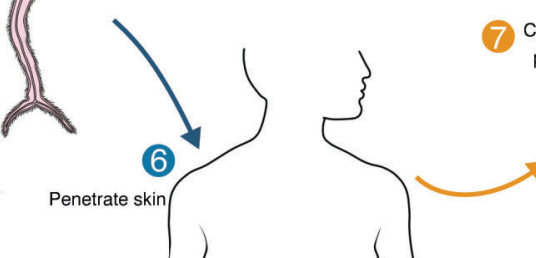
penetration and become schistosomula
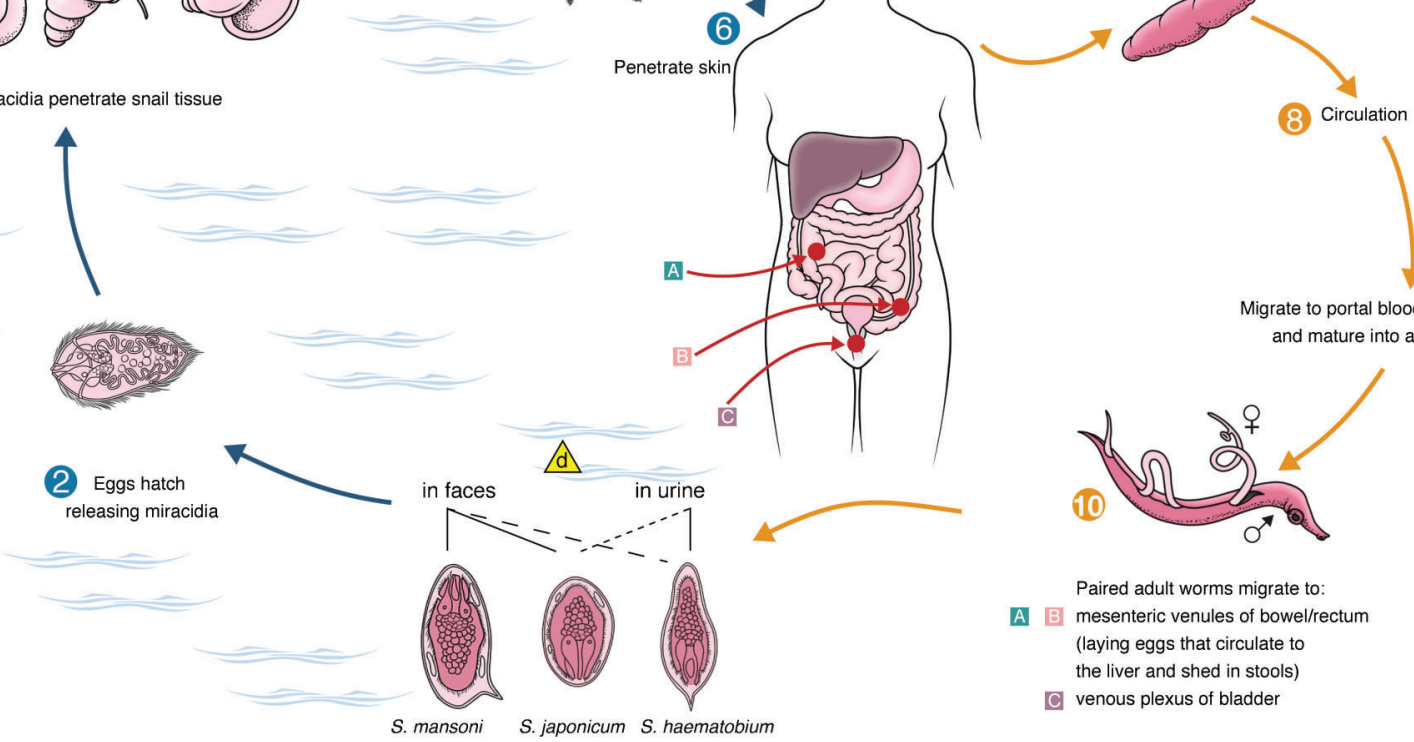

B

A

(1)

Fig 2. Life cycle of S. mansoni, S. japonicum, and S. haematobium. Adapted from McManus DP et al. ${ }^{6}$

shunts (DSRS), and classic splenorenal shunt (SRS). SRS is associated with higher rates of death and hepatic encephalopathy than both EGDS and DSRS. However, high rates of variceal rebleeding after EGDS (27\%) and incidences of encephalopathy after DSRS $(19 \%)$ have been reported. ${ }^{25}$

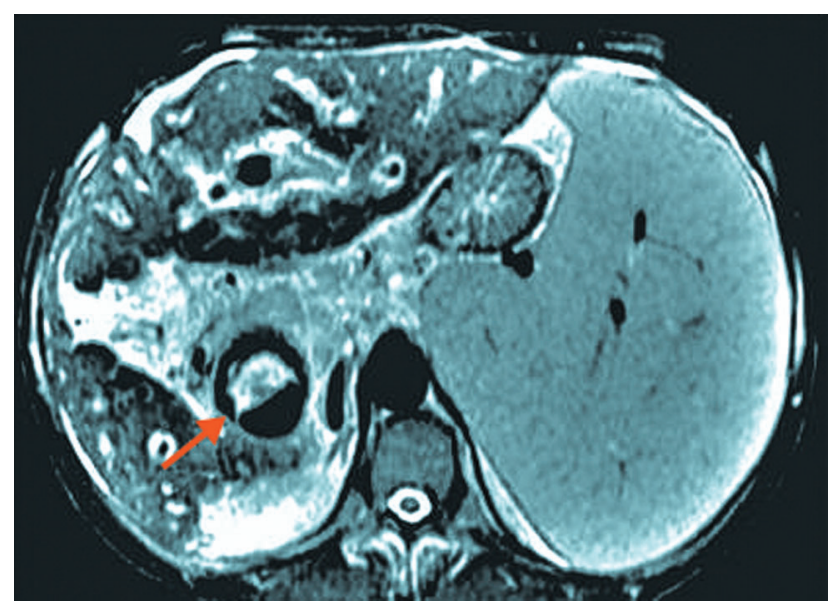

Fig 3. Liver fibrosis in hepatosplenic schistosomiasis. Adapted from Lambertucci JR. ${ }^{32}$
Following diagnosis of schistomiasis, praziquantel should be administered as soon as possible. Praziquantel can safely be given to pregnant and lactating women, and it decreases the disease burden and improves pregnancy and fetal outcomes. The implementation of programs targeting women during reproductive years will improve their reproductive life and overall well-being. ${ }^{26}$

\section{Morbidity and mortality}

Acute schistosomiasis is associated with a mortality rate as high as $25 \%$ in some studies. Although most individuals with chronic schistosomiasis have few or no symptoms, significant morbidity can develop. Complaints are difficult to quantitate because of the geographic distribution of schistosome infection in developing nations and the frequency of co-morbidity with other conditions, such as viral hepatitis. Hepatosplenic disease with portal hypertension is the most common longterm serious complication, followed by cardiopulmonary involvement, obstructive nephropathy, bacteremia, and malignancy. Female genital infection can contribute to pregnancy complications, including reports of related ectopic pregnancy. Urogenital schistosomiasis is considered to be a risk factor for HIV infection, especially in women. ${ }^{27}$ End-stage hepatosplenic disease with variceal bleeding, pulmonary hypertension with cor pulmonale, and central nervous system 
disease are associated with high mortality rates; and carcinoma of the liver and gallbladder may cause death. Although effective anti-helminthic treatment exists, it may not be readily available in endemic areas and may not reverse existing fibrosis. Re-infection is extremely common in people who live in or return to endemic areas. Therefore, repetitive treatment is necessary to prevent disease progression in this situation. 27

\section{Anti-schistosomal vaccination}

Researchers are optimistic in the successful development and implementation of an anti-schistosomal vaccine. The greatest advances in schistosomiasis vaccine technology have been in the integrated genomic and proteomic study on antigen selection. The apical membrane proteins expressed on the surface of the schistosomulum and the adult worm are logical vaccine targets, and there is some data available in support of this hypothesis. ${ }^{28,29}$ Another putative target, although not yet explored, is the mRNAs encoding novel, putatively secreted proteins with no known homologues that are lodged in the tegument membrane.There are very few schistosomiasis vaccine trials that target proteins completely unique to schistosomes and do not share sequence identity with any other proteins. ${ }^{30,31}$

Anti-schistosome vaccines should not be considered as a panacea. It is just one component of integrated schistosomiasis control programs that include chemotherapy and health education. Although controversial, praziquantal resistance is a major concern, and the necessity for vaccines is now more important than ever. ${ }^{6}$

\section{Conclusions}

HSS is a highly prevalent and preventable parasitic disease in which damage to the hepatobiliary system is mediated by the host immune response. Knowledge of the immunopathogenesis and clinical aspects of the infestation may lead to the development of new treatment strategies and improved disease prognosis.

\section{Conflict of interest}

None

\section{Author contributions}

Writing the review (YS, NS, EA).

\section{References}

[1] http://www.who.int/wer, accessed Sept 2010

[2] Chistulo L, Loverde P, Engels D. Schistosomiasis. Nat Rev Microbiol 2004;2: 12-13.

[3] Gryseels B, Polman K, Clerinx J, Kestens L. Human schistosomiasis. Lancet 2006;368:1106-1118. doi: 10.1016/S0140-6736(06)69440-3.

[4] Nour NM. Schistosomiasis: Health Effects on Women. Rev Obstet Gynecol 2010;3:28-32.

[5] Warren KS. Schistosomiasis: Host-Pathogen Biology. Rev Infect Dis 1982; 4 : 771-775. doi: 10.1093/4.4.771.

[6] McManus DP, Loukas A. Current Status of Vaccines for Schistoso miasis. Clin Microbiol Rev 2008;21:225-242. doi: 10.1128/CMR.00046-07

[7] Weinstock JV. The pathogenesis of granulomatous inflammation and organ injury in schistosomiasis: interactions between the schistosome ova and the host. Immunol Invest 1992;21:455-475. doi: 10.3109/ 08820139209069384

[8] El-Khoby T, Galal N, Fenwick A. The USAID/Government of Egypt's Schistosomiasis Research Project (SRP). Parasitol Today 1998;14:92-96. doi: 10.1016/S0169-4758(97)01206-4.

[9] Warren KS, Domingo EO. Granuloma formation around Schistosoma mansoni, S. haemtobium and S. japonicum eggs. Size and rate of development, cellular composition, cross-sensitivity, and rate of egg destruction. Am J Trop Med Hyg 1970;19:292-304.

[10] Stadecker MJ. The development of granulomas in schistosomiasis: Genetic backgrounds, regulatory pathways, and specific egg antigen responses that influence the magnitude of disease. Microbes Infect 1999;1:505-510. doi: 10.1016/S1286-4579(99)80089-6.

[11] Pearce EJ, Caspar P, Grzych JM, Lewis FA, Sher A. Down- regulation of Th-1 cytokine production accompanies induction of Th-2 responses by a parasite helminth Schistosoma mansoni. J Exp Med 1991;173:159-166. doi: 10.1084/jem.173.1.159.

[12] Rihet P, Demeure CE, Bourgois A, Prata A, Dessein AJ. Evidence for an association between human resistance to Schistosoma mansoni and high anti-larval IgE levels. Eur J Immunol 1991;21:2679-2686.

[13] Dunne DW, Grabowska AM, Fulford AJ, Butterworth AE, Sturrock RF, Koech D, et al Human antibody responses to Schistosoma mansoni: The influence of epitopes shared between different life-cycle stages on the response to the schistosomulum. Eur J Immunol 1988;18:123-131.

[14] Strickland GT, Abdel-Warib ME Schistosomiasis. In Strickland GT (ed): Hunter's Tropical Medicine, ed 7. Philadelphia, WB Saunders, 1991:781-802.

[15] Malaquias LC, Falcao PL, Silveira AM, Gazzinelli G, Prata A, Coffman RL, et al. Cytokine regulation of human immune response to Schistosoma mnsoni: Analysis of the role of IL-4, IL-5 and IL-10 on peripheral blood mononuclear cell responses. Scand J Immunol 1997;46:393-398. doi: 10.1046/j.13653083.1997.d01-136.x.

[16] Andrade ZA. Pathology of human schistosomiasis. Mem Inst Oswaldo Cruz 1987;82(suppl 4):17-23. doi: 10.1590/S0074-02761987000800005.

[17] Sheta EA, El Saadany S. Schistosomiasis. Tanta Medical Sciences Journa 2006;1:1-10.

[18] Andrade ZA, Peixoto E, Guerret S, Grimaud JA. Hepatic connective tissue changes in hepatosplenic schistosomiasis. Hum Pathol 1992;23:566-573. doi: 10.1016/0046-8177(92)90135-P.

[19] Ghaffar YA, Fattah SA, Kamal M, Badr RM, Mahomed FF, Strickland GT. The impact of endemic schistosomiasis on acute viral hepatitis. Am J Trop Med Hyg 1991;45:743-750.

[20] Darwish MA, Raouf TA, Rushdy P, Constantine NT, Rao MR, Edelman R. Risk factors associated with a high seroprevalence of hepatitis $C$ virus infection in Egyptian blood donors. Am J Trop Med Hyg 1993;49:440-447.

[21] Larouze B, Dazza MC, Gaudebout C, Habib M, Elamy M, Cline B. Absence of relationship between Schistosoma mansoni and hepatitis B virus infection in the Qalyub Govemate, Egypt. Ann Trop Med Parasitol 1987;81:373-375.

[22] Bassily S, Strickland GT, Abdel-Wahab MF, Esmat GE, Narooz S, el-Masry NA, et al. Efficacy of hepatitis B vaccination in primary school children from a village endemic for Schistosoma mansoni. J Infect Dis 1992;166:265-268. doi: 10.1093/infdis/166.2.265.

[23] Karanja DMS, Colley DG, Nahlen BL, Ouma JH, Secor WE. Studies on schistosomiasis in western Kenya: Evidence for immune-facilitated excretion of schistosome eggs from patients with Schistosoma mansoni and human immunodeficiency virus coinfections. Am J Trop Med Hyg 1997;56:515-521.

[24] Ishii A, Matsuoka $H$, Aji T, Ohta N, Arimoto S, Wataya $Y$, et al. Parasite infection and cancer with special emphasis on Schistosoma juponicum infections (Trematoda). A review. Mutat Res 1994;305:273-281. doi: 10.1016/0027-5107(94)90247-X.

[25] Vennervald B], Dunne DW. Morbidity in schistosomiasis: an update. Curr Opin Infect Dis 2004;17:439-447. doi: 10.1097/00001432-200410000-00009.

[26] Savioli L, Crompton DW, Neira M. Use of anthelminthic drugs during pregnancy. Am J Obstet Gynecol 2003;188:5-6. doi: 10.1067/mob.2003.78.

[27] http://www.who.int/mediacentre/factsheets/fs115/en/, accessed Oct 2010.

[28] Cardoso FC, Pacífico RN, Mortara RA, Oliveira SC. Human antibody responses of patients living in endemic areas for schistosomiasis to the tegumenta protein Sm29 identified through genomic studies. Clin Exp Immunol 2006 144:382-391. doi: 10.1111/j.1365-2249.2006.03081.x.

[29] Loukas A, Tran M, Pearson MS. Schistosome membrane proteins as vaccines. Int J Parasitol 2007;37:257-263. doi: 10.1016/j.ijpara.2006.12.001.

[30] Tran, MH, Pearson MS, Bethony JM, Smyth DJ, Jones MK, Duke M, et al. Tetraspanins on the surface of Schistosoma mansoni are protective antigens against schistosomiasis. Nat Med 2006;12:835-840. doi: 10.1038/nm1430.

[31] Braschi S, Curwen RS, Ashton PD, Verjovski-Almeida S, Wilson A. The tegument surface membranes of the human blood parasite Schistosoma mansoni: a proteomic analysis after differential extraction. Proteomics 2006; 6:1471-1482. doi: 10.1002/pmic.200500368.

[32] Lambertucci JR. Revisiting the concept of hepatosplenic schistosomiasis and its challenges using traditional and new tools. Rev Soc Bras Med Trop 2014 47:130-136. doi: 10.1590/0037-8682-0186-2013. 